\title{
LTE Amplify and Forward Relaying for Indoor Coverage Extension
}

\author{
Thomas Wirth, Lars Thiele, Thomas Haustein \\ Fraunhofer Institute for Telecommunications \\ Heinrich Hertz Institute \\ Einsteinufer 37, 10587 Berlin, Germany \\ thomas.wirth@hhi.fraunhofer.de
}

\author{
Oliver Braz, Jörg Stefanik \\ Andrew Wireless Systems GmbH \\ Industriering 10 \\ 86675 Buchdorf, Germany \\ oliver.braz@commscope.com
}

\begin{abstract}
In many countries more than $70 \%$ of the traffic on cellular networks originates or terminates inside buildings. Driven by an increasing demand for mobile data services, the iPhone effect, this number is expected to rise even higher within the next years [1]. When rolling out new systems addressing high speed data needs, e.g. LTE or HSPA+, a cost effective solution is explored by extending the outdoor macro coverage to in-side. In order to reduce the building penetration path loss, a relay can be used in an amplify and forward (AF) or decode and forward (DF) operation for improving the signal quality. The benefits of a DF relay on the achievable LTE downlink indoor coverage extension has been demonstrated in [2]. Implementing the DF relay, however, adds complexity to the system and decreases overall air-interface capacity due to the in-band backhauling. The question then arises as to what level of performance can be expected from a straight forward AF system. This paper reports on first measurement results from deploying a standard optical distributed antenna system (DAS) for the LTE downlink at $2.6 \mathrm{GHz}$ in a LTE real-time testbed. Field trials are performed in a single cell single user setup within an indoor office scenario with various MIMO antenna installations. Results show that the full data throughput of $150 \mathrm{Mbps}$ can be transferred from the macro cell to the inside of the building.
\end{abstract}

Index Terms - amplify and forward (AF) relaying, LTE field trials, distributed antenna system (DAS), indoor coverage

\section{INTRODUCTION}

In the last few years the demand for higher network capacity and performance in wireless services has been growing exponentially. Several options such as increasing the channel bandwidth, optimizing the modulation and coding schemes or even implementing a code-multiplexing system have improved data throughput. Now, spectral efficiency is being increased through a multiple-input multiple-output (MIMO) implementation, where signals are space-multiplexed with antenna arrays. Significant link capacity enhancement in the LTE downlink, exceeding $150 \mathrm{Mbps}$ in a 2x2 MIMO system, has been successfully demonstrated in single-cell scenarios in [3] and [4]. New challenges especially arise in fast deployment strategies of initial LTE infrastructures. Here, LTE-Advanced relaying, refer to [2] and [5], shows promising results and is a hot topic in current standardization for LTE-Advanced, see [6] and [7]. Simpler amplify and forward (AF) relays could minimize deployment costs and optimize coverage especially in indoor scenarios. Increasing indoor coverage and increasing capacity in hotspot areas will become the key factor during initial LTE deployment.

\section{Berlin LTE-AdVANCED TESTBED}

For the experiments we used the Berlin LTE-Advanced Testbed, which was installed in the center of Berlin throughout 2008. This 3G-LTE testbed consists of three base station sites with nine sectors. In the following we will refer to base station as enhanced node B (eNB). The user terminal will be referred to as user equipment (UE). The measurements at the terminal were performed in the building of the Heinrich Hertz Institute (HHI), which is in approximately $500 \mathrm{~m}$ distance from the eNB site. The measurements are limited to an isolated single-sector scenario without multi-cell interference from other eNBs. The eNB antennas are mounted on an antenna pole on top of the building, $85 \mathrm{~m}$ above ground. The carrier frequency was $2.6 \mathrm{GHz}$ which is within the UMTS extension band. The testbed incorporates LTE key features such as adaptive bit-loading, frequency dependent scheduling in $20 \mathrm{MHz}$ bandwidth based on closed-loop feedback. The link adaptation has a $10 \mathrm{~ms}$ duty cycle, considering channel quality information (CQI) and preferred matrix index (PMI) feedback. According to the PMI feedback, the eNB scheduler applies adaptive MIMO mode selection for $2 \times 2$ MIMO supporting SIMO, spatial multiplexing or SDMA. The cyclic prefix (CP) is set to $4.7 \mu \mathrm{s}$. The maximum achievable throughput on the physical layer (PHY layer) reaches approximately $150 \mathrm{Mbps}$. On IP layer, the LTE system realizes a low round-trip delay of only $8 \mathrm{~ms}$.

The optical signal distribution has been realized by an Andrew/CommScope ION-M based MIMO prototype system. As in-building coverage antennas, the CELLMAX-O-CPUSE has been used.

\section{RELAYING CONCEPT}

The measurements focus on the amplify and forward (AF) relaying concept. The basic concept and its pros and cons will be compared to the decode and forward (DF) concept in more detail below.

\section{A. Decode and Forward Relaying}

In the DF mode, the signal received from the source terminal is demodulated and decoded before retransmission. 
The decision process required for the signal's constellation remapping removes the overlaid received noise components and thus the overall noise radiated into the cell is reduced [8]. By using sophisticated HARQ and multi-hop schemes, the overall network performance is enhanced [9]. However, this benefit is not for free. The DF relay increases complexity and it should be noted that the signal processing required cannot be performed within one symbol time length. As such, dedicated time slots for the eNB - relay communication must be introduced. Since time slots used for backhauling tasks are not available for the eNB - UE data exchange, the overall network air interface capacity is reduced [2].

\section{B. Amplify and Forward Relaying}

In the AF mode, the relay terminal simply amplifies and retransmits the signal received from the source terminal. Thus, the signal received at the relay terminal is corrupted by fading and additive noise. For low signal to noise ratio (SNR) the relay's noise figure becomes significant and system impacting and can result in decreased channel capacity [8]. However, in-building systems usually do not suffer from low SNR ratio because providing homogeneously sufficient signal power is simply a question of appropriate indoor radio planning. The basic lineup of the optical distributed antenna system is shown in Fig. 1.

For the eNB - relay link in the test setup, two identical $45^{\circ}$ cross-polarized base station antennas with $18 \mathrm{dBi}$ gain have been used. This results in a downlink donor signal level of approximately $-30 \mathrm{dBm}$. Before the electro-optical conversion both single frequency MIMO signals are frequency shifted and filtered. The power of the radiated signal can be level adjusted to compensate for cable loss of the passive antenna portion of the system. This gain is in the range of $30-50 \mathrm{~dB}$. After transmission via a $100 \mathrm{~m}$ optical cable both MIMO signals are re-converted to their original frequencies. To minimize the inbuilding coverage contribution from the macro cell the down tilt of the eNB antenna had been set to $0^{\circ}$. The resulting poor indoor coverage and thus low data throughput on the 6th floor is shown in Fig. 2. This scenario is realistic since the down tilt will be optimized to a specific value in a order to achieve the maximum coverage or capacity, e.g. on the ground floor or for an outdoor scenario. Therefore, there are always areas with reduced capacity in a multi-storied building. In addition, solar protected windows as found in modern office buildings will reduce the received signal strength by approximately 20 $30 \mathrm{~dB}$.

\section{Measurement Scenarios}

For an in-building distributed antenna system (DAS) system, the main design criteria is given by the installation costs involved with different antenna configurations. For upgrading to a MIMO distribution system, it would be most cost effective to reuse the existing single input single output (SISO) $3 \mathrm{G}$ system. This avoids major construction activities like opening up the ceiling to bring in a second cable run for the MIMO signal path. However, it should be evaluated whether a simple

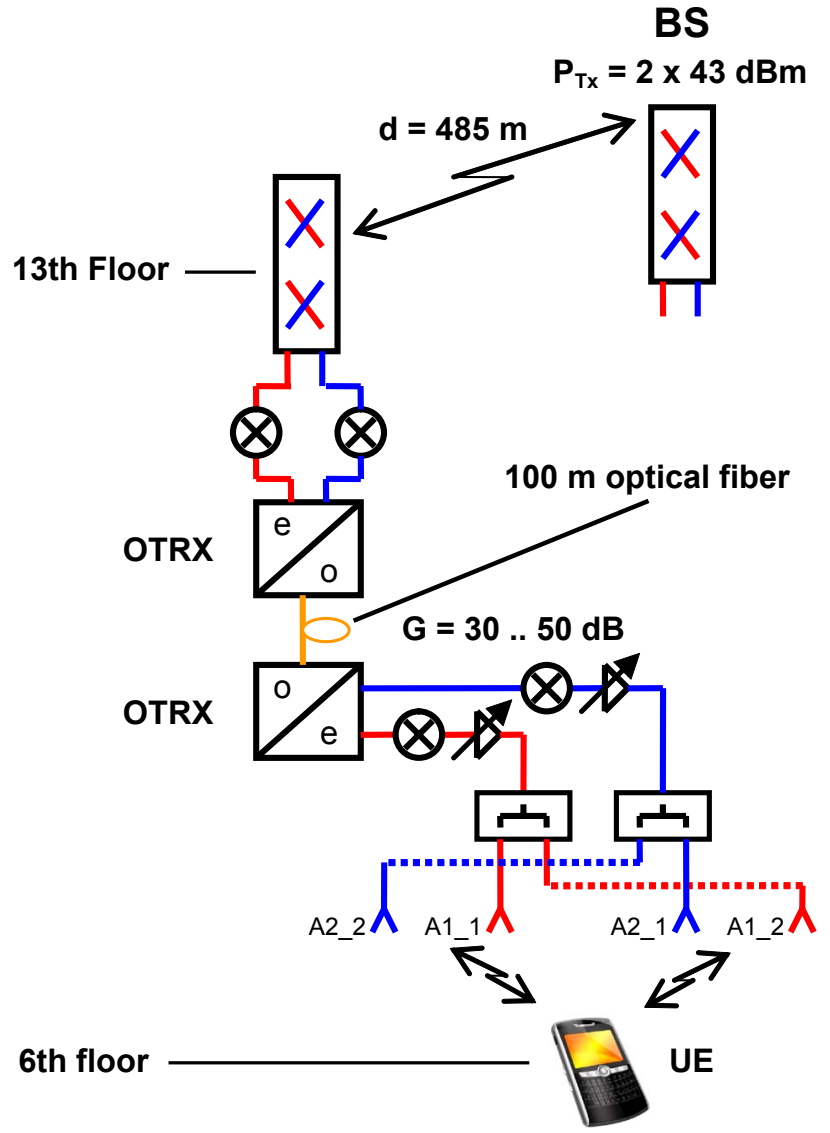

Fig. 1. Optical AF distributed antenna system (DAS) concept: BS - Base Station, UE - User Equipment, OTRX - Optical Transceiver, An_m - MIMO stream $\mathrm{n}$ at coverage antenna $\mathrm{m}$.

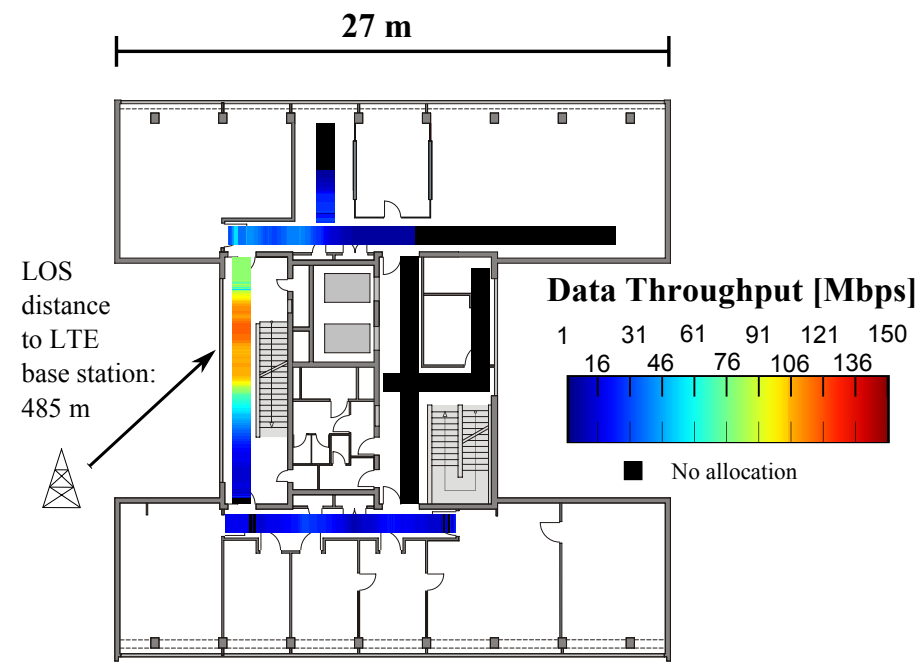

Fig. 2. Graphical representation of the in-building coverage without relay measured on the 6th floor. 


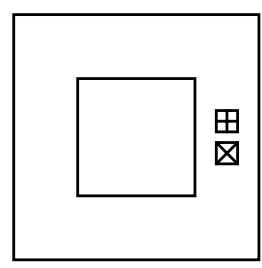

A.

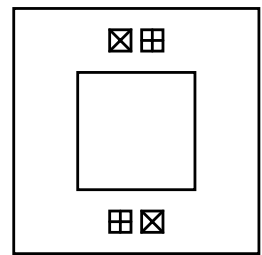

C.

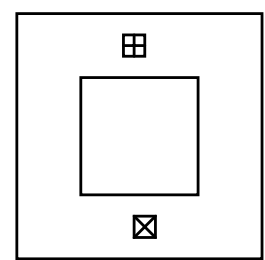

B.

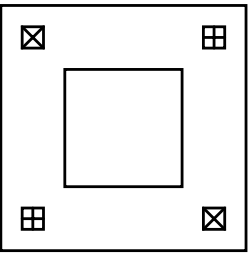

D.
Fig. 3. Schematic floor plans of the different antenna placements in the measurement scenarios.

reconfiguration of the passive distribution system will be sufficient from a performance standpoint. For this reason four different antenna configurations, as shown in Fig. 3, have been investigated.

It shall be noted that as according to [10], no performance differences between cross-polarized or co-polarized antenna arrangements have been observed indoors in the investigated corridor environment. However, for large hallways or conference rooms this may look different.

\section{A. Co-located Antenna Pair}

A straight forward approach to extend indoor coverage is to place antennas where there is no penetration from the outside macro cell. As shown in the investigation performed within this trial, Fig. 2, a certain amount of coverage is provided through the windows facing direct line of sight (LOS) to the eNB. The first location for an additional pair of antennas is the stairway on the opposite part of the building.

\section{B. Distributed Antennas}

A $2 \mathrm{G}$ or $3 \mathrm{G}$ antenna installation would most likely benefit from the corridor effect and therefore would have an installed antenna on the opposite side of the floor. Since cable runs must have been installed for this configuration already, the upgrade to a MIMO system might be possible by simply connecting the system at the distribution point.

\section{Distributed paired antennas}

Some benefit from the installed equipment can be achieved by reusing the same antenna locations, and by installing a second antenna close to the position of the SISO antennas. However, the second MIMO antenna signal has to be distributed to this location. This can be achieved by installing an additional cable run or by reusing the existing cabling and frequency shifting the second path over the same passive media.

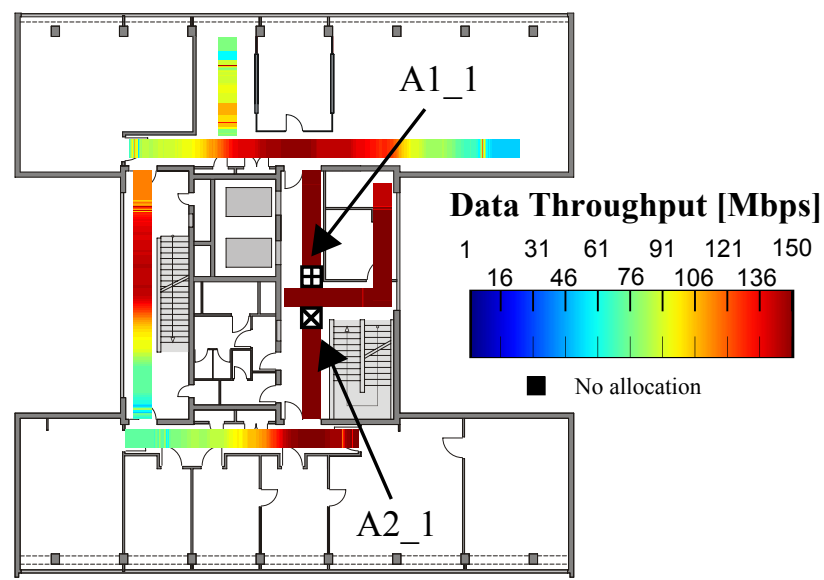

Fig. 4. Scenario A: Graphical representation of the in-building coverage with co-located antennas.

\section{Interleaved distributed antennas}

The most elaborated alternative is to install two additional interleaved antennas. This would facilitate a change in the position of the existing antenna locations as well. From a MIMO performance point of view the introduced rich indoor scattering is supposed to be the optimal solution.

\section{E. Recording of Measurement Statistics}

The UE allows real-time recording of bit error rates (BERs), per antenna received power, and PHY layer throughput. Other statistics that can be recorded are e.g. broadband channel coefficients. During the measurements, the eNB transmits with full buffer to maximize the users sum rate, which is recorded on the LTE PHY layer with code-rate 0.5. The maximum achievable throughput on the LTE PHY is approximately 150 Mbps. The link adaptation is configured with a BER target of $\leq 0.02$. The terminal takes the BER target into account during calculation of the CQI and PMI feedback vectors. In addition to throughput data, the received power statistics can be recorded. These show the received power in $\mathrm{dBm}$ averaged over the two receive antennas of the mobile terminal.

\section{INDOOR RESULTS}

In the following paragraph the measurement results achieved by deploying the above mentioned antenna configurations are discussed. For comparison, consider the indoor coverage situation without the DAS as shown in Fig. 2.

\section{A. Co-located Antenna Pairs}

As illustrated in Fig. 4 the coverage in the opposite side of the building from the eNB can be improved significantly. It has to be noted that for these trials the direct eNB indoor coverage has been degraded by reducing the down tilt of the base station antenna. The regular LOS in-building coverage is documented in [2]. Under regular conditions, with the above mentioned antenna deployment, a satisfactory in-building coverage has been achieved. 


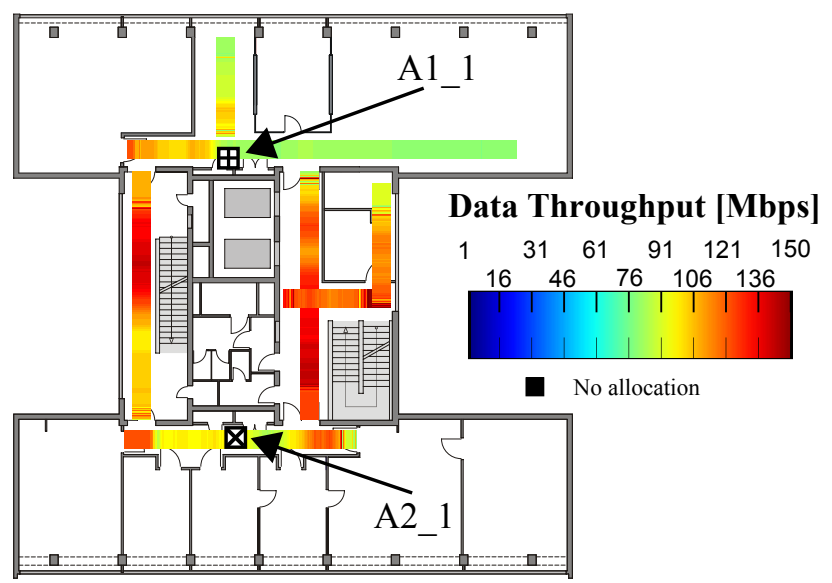

Fig. 5. Scenario B: Graphical representation of the in-building coverage with distributed antennas.

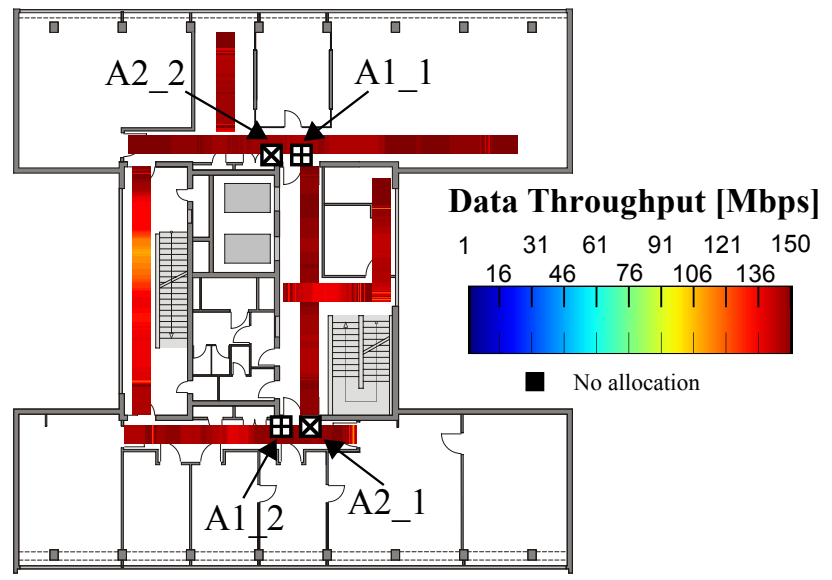

Fig. 6. Scenario C: Graphical representation of the in-building coverage with distributed paired antennas.

\section{B. Distributed Antennas}

The throughput results for the distributed antenna scenario is shown in Fig. 5. Sometimes the UE may switch to single stream operation when the second antenna path loss exceeds a certain value. However, it should be kept in mind that with LTE's advanced collaborative MIMO transmission scheme this situation can be beneficial. In such a scenario, two mobiles, each located close to a different antenna, can share the same frequency allocation simultaneously and so overall network capacity may be increased significantly.

\section{Distributed paired antennas}

In this configuration, the indoor environment in-building coverage is excellent, as shown in Fig. 6. However from a cost-benefit-ratio point of view, the improvement is deemed not worth the cost of a second infrastructure.

\section{Interleaved distributed antennas}

As expected, a superior in-building coverage system can be achieved by implementing an interleaved antenna configuration. As shown in Fig. 7, data throughput of more than $100 \mathrm{Mbps}$ has been achieved over the complete floor.

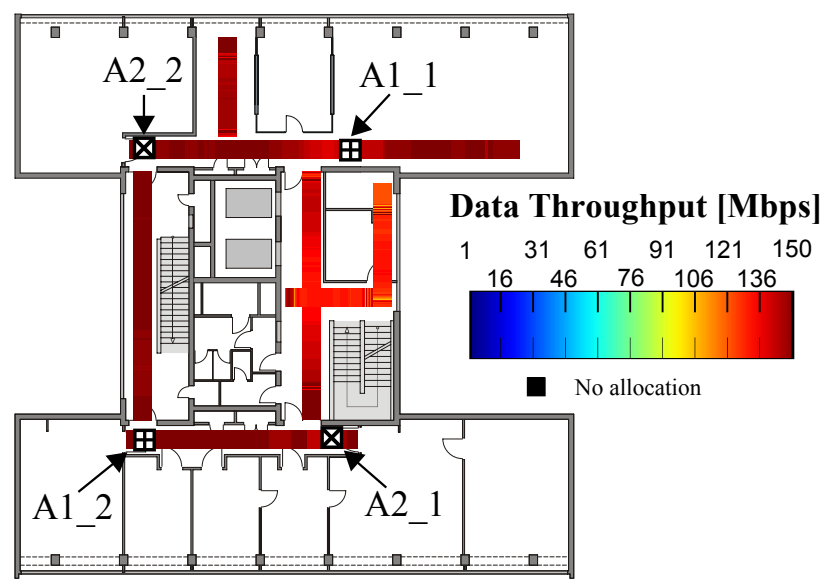

Fig. 7. Scenario D: Graphical representation of the in-building coverage with distributed interleaved antenna pairs. The distance between antennas A1_1 and A2_2, and antennas A1_2 and A2_1 was approximately 9-10 m.

\section{SUMMARY}

The throughput CDFs in Fig. 8 summarize the statistics of the PHY layer throughput along all tracks in each indoor measurement scenario $\mathrm{A}, \mathrm{B}, \mathrm{C}$ and $\mathrm{D}$, and compare results to the case where the terminal is directly connected to the eNB; also refer to Table VI. From these results we can conclude the following:

- Scenario A: Data rates are in $95 \%$ of the cases above $61 \mathrm{Mbps}$.

- Scenario B: Data rates never drop below $75 \mathrm{Mbps}$ with high data rates of $100 \mathrm{Mbps}$ for the median.

- Scenarios C and D: Data rates are always above 100 Mbps. Both antenna distributions show a quite similar performance. In general in scenarios $\mathrm{C}$ and $\mathrm{D}$, the eNB can utilize the second transmit stream and the UE benefits from cross-polarization multiplexing. This obviously results in a better performance as compared to scenarios $\mathrm{A}$ and $\mathrm{B}$ which is shown by higher data rates and steeper CDFs.

- Direct link: Data rates show a high variance of achievable data rates. The direct link shows a very high outage of more than $40 \%$. The high data rates are only available in LOS to the eNB.

Comparing the direct link to the AF link, there is no outage in the $\mathrm{AF}$ deployment. AF relaying shows a significantly reduced variance in data rate and UEs always benefit from the second stream resulting from cross-polarization multiplexing.

\begin{tabular}{|c|c|c|c|}
\hline Scenario & 5\% Quantile & Mean & Standard Deviation \\
\hline \hline direct link & 6 & 22 & 33 \\
A & 61 & 119 & 33 \\
B & 75 & 102 & 23 \\
C & 112 & 141 & 13 \\
D & 117 & 141 & 15 \\
\hline
\end{tabular}

TABLE I

Sum throughput STATISTICS OF LTE's PHY-LAYER IN MBPS 


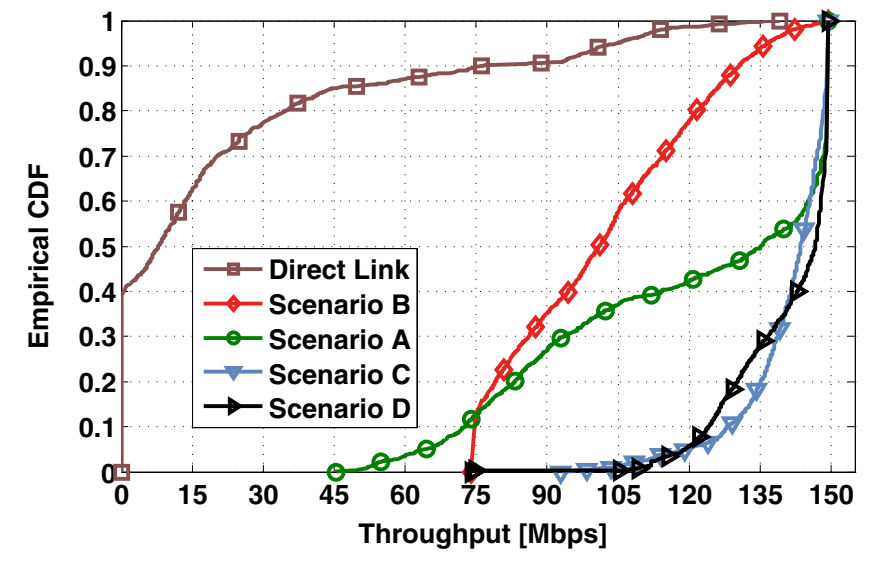

Fig. 8. PHY-Layer throughput statistics in Mbps. Statistics for the direct link to the base station, see Fig. 2, are compared with results from the AF deployments as defined in scenarios A-D.

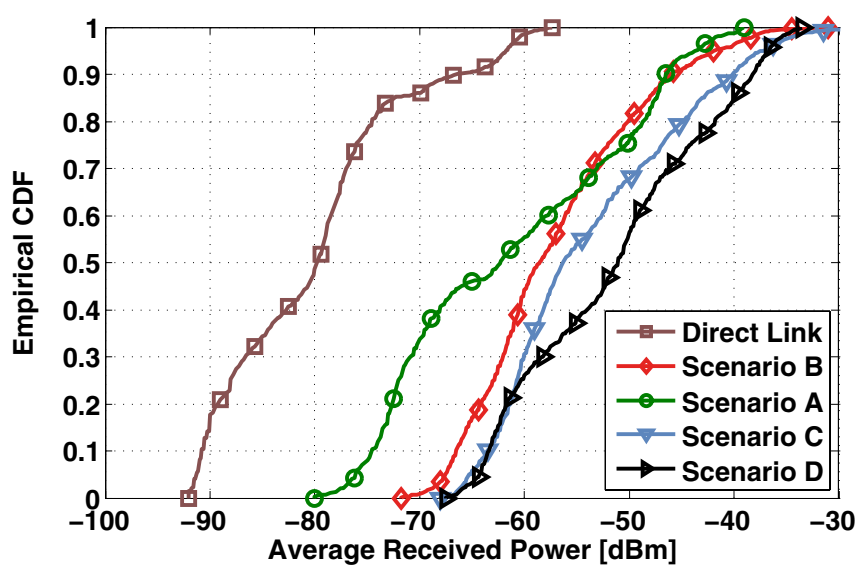

Fig. 9. The cdfs show the average received power statistics. Direct link statistics are compared with results from the AF deployments as defined in scenarios A-D.

Finally, Fig. 9 shows the average received power statistics experienced by the UE for each measurement scenario. The indoor DAS deployment increases the indoor received power by $20-30 \mathrm{dBm}$ for the median. The DAS deployments in scenarios A-D have a median received power comparable with the peak received power of approximately $-60 \mathrm{dBm}$ achieved in the direct link connection. In the direct link, the highest received power is only reached in case the terminal is in LOS to the eNB. This is only the case on a small part of one measurement track on the left part of the building. The mean received power in all DAS deployment scenarios lies above $-62 \mathrm{dBm}$. At this operating point the system can utilize more resources in MIMO mode than in a single stream transmission, which was shown in [3]. Scenarios C and D obviously show the best performance with a power gain of more than $3 \mathrm{~dB}$ as compared to the antenna setups in scenarios A and B.

\section{CONCLUSION}

Despite the limited isolation between the cross-polarized base station and donor antenna elements, the introduced cor- relation, LTE's spatial multiplexing scheme seems to be very robust. As this paper shows, the MIMO coded signal can be transmitted without any performance degradation in good LOS conditions. The interleaved distributed antenna configuration is the most advantageous deployment. At a minimal upgrade cost, it provides exceptional data rates and the LTE precoding supports the transmission of two separate spatial data streams at two different antennas. It is therefore possible to fully exploit the rich scattering that exists in most in-building environments and supply users with high data rates even in difficult deployment scenarios.

From a single user data throughput point of view, best results in our small size corridor environment have been achieved by installing the antennas in an interleaved antenna arrangement. For the re-use of existing $2 \mathrm{G}$ or $3 \mathrm{G}$ antenna installations, the collaborative MIMO implementation foreseen in LTE-Advanced seems to be beneficial. By sharing the same frequency resource at different antenna locations the overall network capacity and coverage can be increased. Future investigations will focus on the feeder link and the impact of multi-cell interference. In this case, the impact of interference amplification has to be analyzed which is a topic of ongoing research.

\section{ACKNOWLEDGMENT}

The base station sites of the Berlin LTE-Advanced Testbed are jointly operated by the Heinrich Hertz Institute and the Deutsche Telekom. The testbed was funded by the Bundesministerium für Bildung und Forschung (BMBF) under grant 01BU0631.

\section{REFERENCES}

[1] ABIresearch, "In-building wireless systems - passive and active das, repeaters, picocells, and femtocells," 2009. [Online]. Available: www.abiresearch.com

[2] V. Venkatkumar, T. Wirth, T. Haustein, and E. Schulz, "Relaying in long term evolution: Indoor full frequency reuse," in Proc. European Wireless (EW), Aalborg, Denmark, May 2009.

[3] T. Haustein, V. Venkatkumar, J. Eichinger, E. Schulz, T. Wirth, V. Jungnickel, A. Forck, S. Wahls, C. Juchems, F. Luhn, and R. Zavrtak, "Measurements of multi-antenna gains using a 3GPP-LTE air interface in typical indoor and outdoor scenarios," in Proc. European Wireless (EW), Prague, Czech Republic, Jun. 2008.

[4] T. Wirth, V. Jungnickel, A. Forck, S. Wahls, H. Gaebler, T. Haustein, J. Eichinger, D. Monge, E. Schulz, C. Juchems, F. Luhn, and R. Zavrtak, "Realtime multi-user multi-antenna downlink measurements," Proc. IEEE Wireless Communications and Networking Conference (WCNC), Mar. 2008.

[5] T. Wirth, V. Venkatkumar, T. Haustein, E. Schulz, and R. Halfmann, "LTE-Advanced relaying for outdoor range extension," in VTC2009-Fall, Anchorage, USA, Sep. 2009.

[6] 3GPP R1-090379 Ericcson, "Discussion on RAN1 preferences for relaying," Ljubjana, Slovenia, Jan. 2009.

[7] 3GPP R1-082470 Ericcson, "Self backhauling and lower layer relaying," Warsaw, Poland, July 2008.

[8] R. U. Nabar, H. Bölcskei, and F. W. Kneubühler, "Fading relay channels: Performance limits and space-time signal design," IEEE Journal on Selected Areas in Communications, vol. 22, no. 6, pp. 1099-1109, Aug. 2004. [Online]. Available: http://www.nari.ee.ethz.ch/commth/pubs/p/jsac03

[9] Nortel Corp., Transparent Relay for LTE-Advanced FDD, Warsaw, Poland, July 2008.

[10] S. Caban, C. Mehlführer, L. W. Mayer, and M. Rupp, "2x2 MIMO at variable antenna distances," in VTC Spring, 2008, pp. 1311-1315. 